\title{
elsevier_TB_1516
}

\section{The effect of seasonal temperature variation on behaviour and metabolism in the freshwater mussel (Unio tumidus)}

givennameGlenn J. Lurmansurname*

glenn.lurman@ana.unibe.ch

givennameJohanna Waltersurname

givennameHans H. Hoppelersurname

Institute of Anatomy, University of Bern, Baltzerstr. 2, 3012 Bern, Switzerland

${ }^{*}$ Corresponding author.

abstract

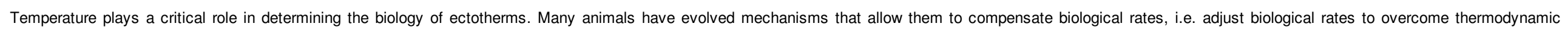

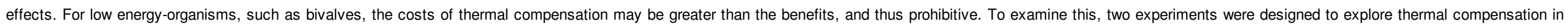

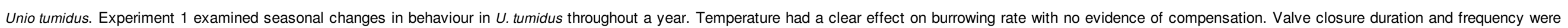

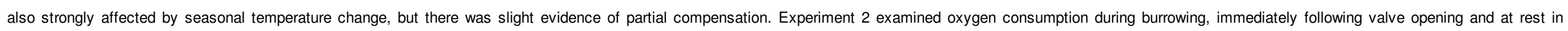

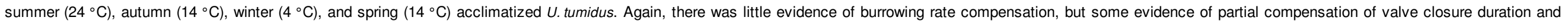

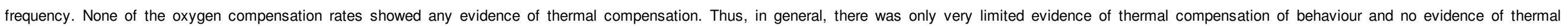

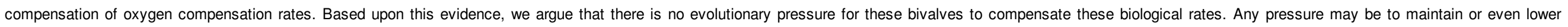

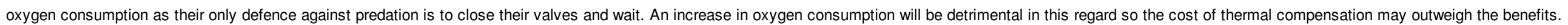

Keywords: Thermal Gomsationcompensation; Acclimation; Oxygen Gonsumptionsumption; Valve Glostreclosure; Burrowing

\section{Introduction}

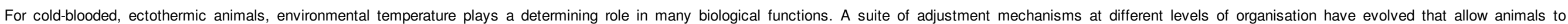

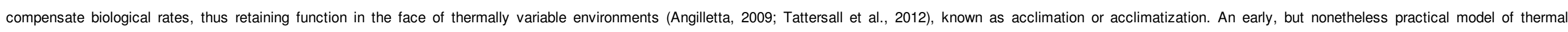

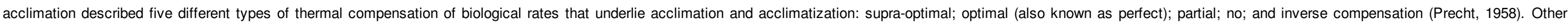

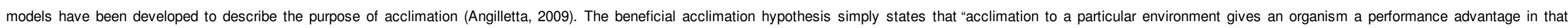

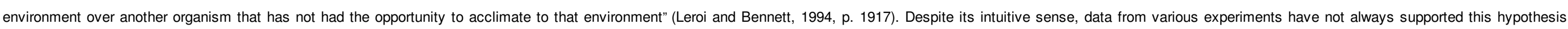

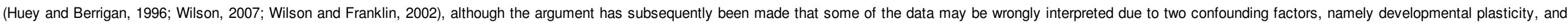
the detrimental effects of holding animals long-term (see Woods and Harrison, 2002 for more details).

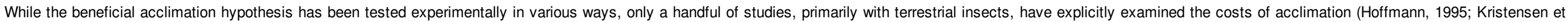

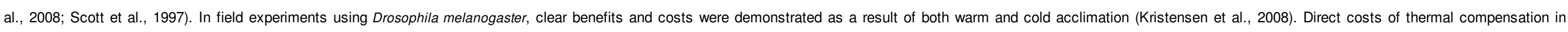

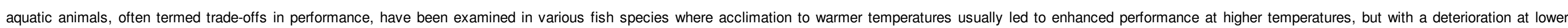

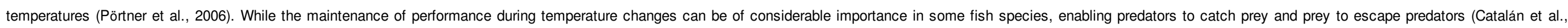

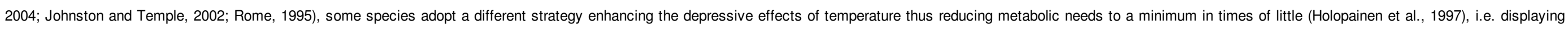
Precht's inverse compensation. 


\section{elsevier_TB_1516}

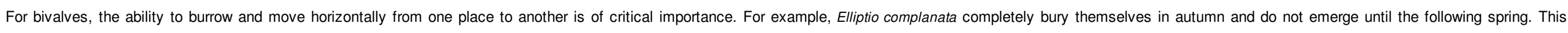

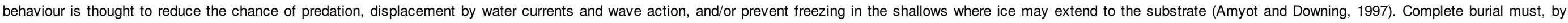

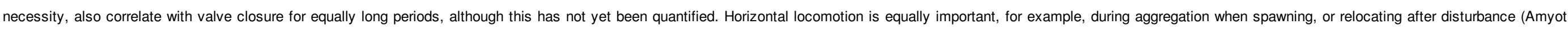

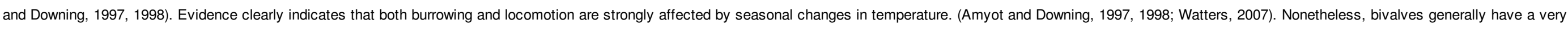

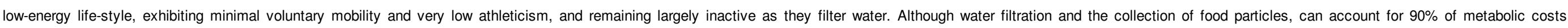

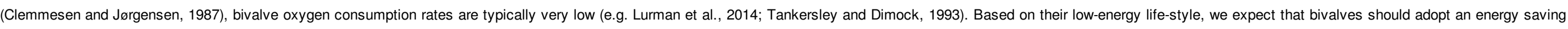
strategy in the face of changing temperatures.

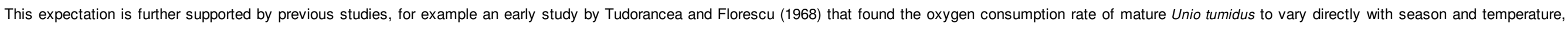

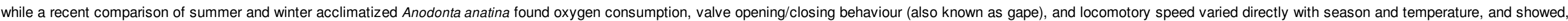

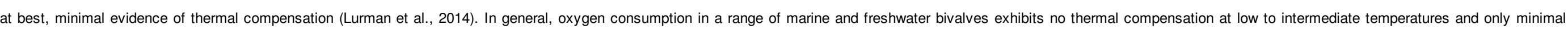

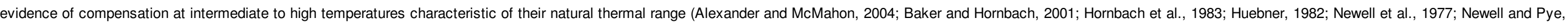
1970; Pernet et al., 2007,2008; Resgalla et al., 2007; Riascos et al., 2012; Tankersley and Dimock, 1993; Widdows, 1973).

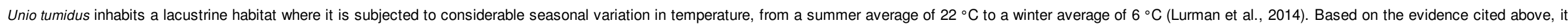

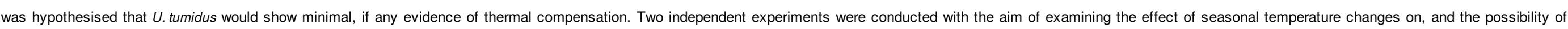

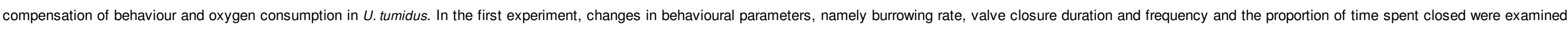
each month throughout one year, with measurements made at a range of temperatures that corresponded to each month.

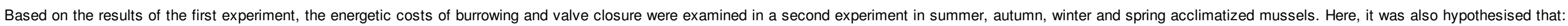

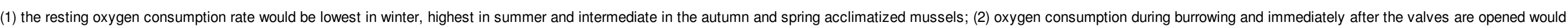

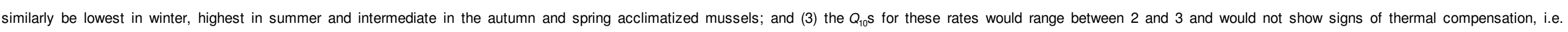
acclimatization.

\section{Methods}

\subsection{Mussel Golleetioncollection and Holdingholding}

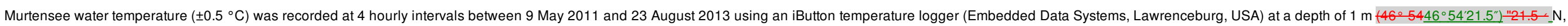
7030.6," E). 70 300.6"E).

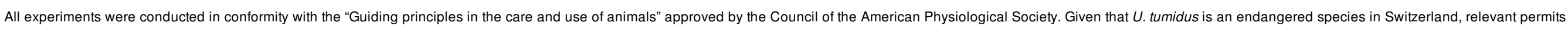
were sought and granted for their use.

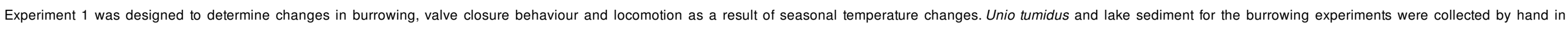

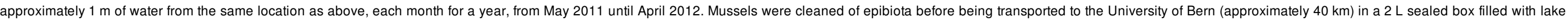

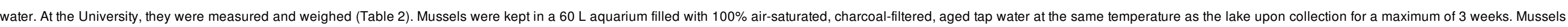

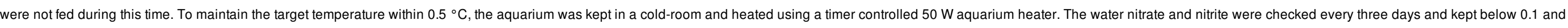
$10 \mathrm{mg} \mathrm{L} \underline{\underline{-1}}$, respectively, with regular water changes.

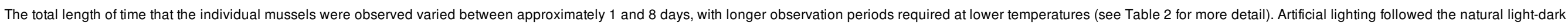
cycle, and a low wattage red light was constantly on to allow behavioural observation at night (see below).

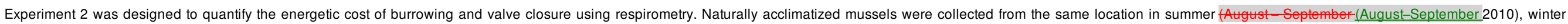

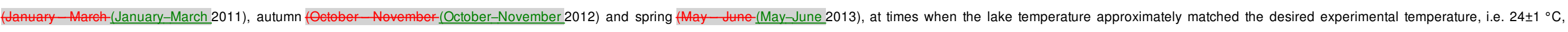




\section{elsevier_TB_1516}

$4 \pm 1{ }^{\circ} \mathrm{C}, 14 \pm 1^{\circ} \mathrm{C}$ and $14 \pm 1 \stackrel{\circ}{\circ} \mathrm{C}$. , respectively. Mussel collection, transportation and holding were as described above for experiment 1.

\subsection{Behavioural Observationobservation}

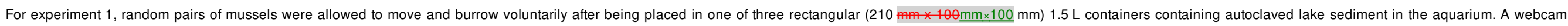

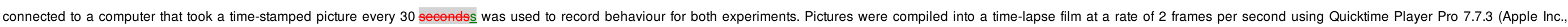
Cupertino, CA). The times at which given activities, namely burrowing, valve closure and opening, as well as locomotory activity, occurred were determined manually.

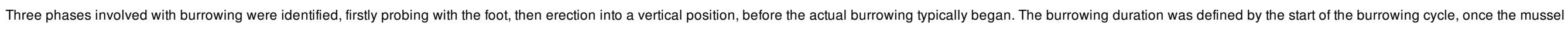
was in a vertical position and the end, i.e. once the mussel was completely inactive. The burrowing rate index was calculated using formula 1, analogous to Peck et al. (2004):

$\mathrm{BRI}=\frac{\text { mussel whole weight }(\mathrm{g})^{1 / 3}}{\text { burrowing duration }(\mathrm{s})} \times 1000$

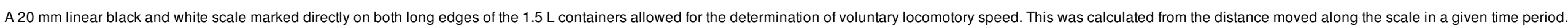

\subsection{Respirometry}

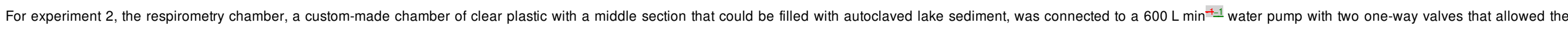

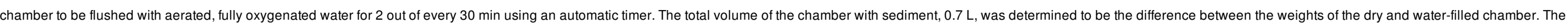

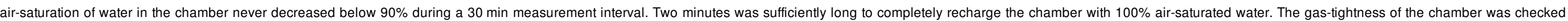

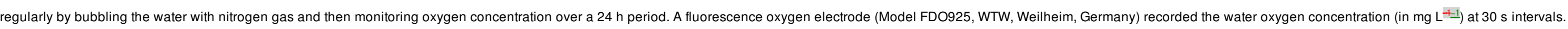
All measurements were automatically temperature compensated.

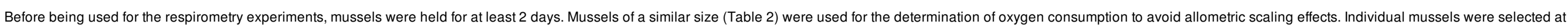

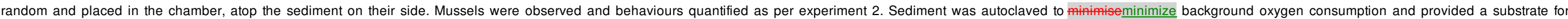

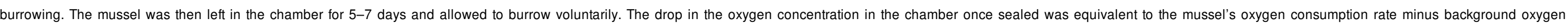
consumption.

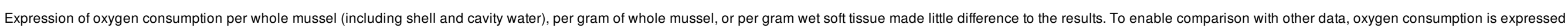

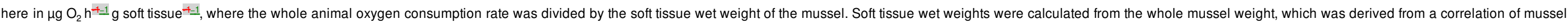

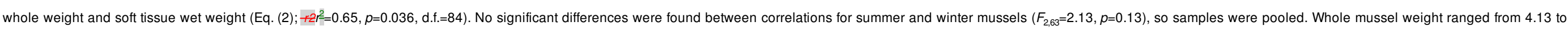
$44.34 \mathrm{~g}$.

Soft tissue wet weight $(\mathrm{g})=(0,185$ whole mussel weight $)+0,73$

\subsection{Statistics}

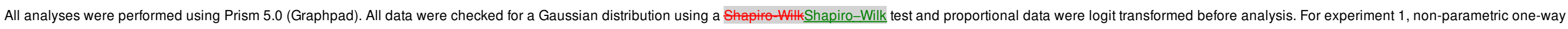

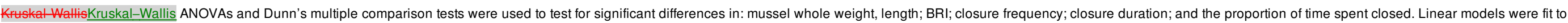

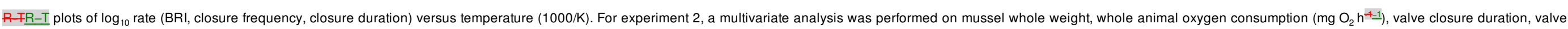

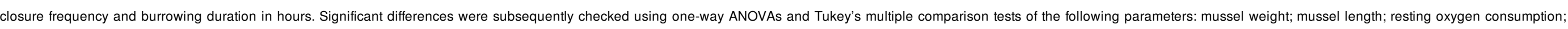

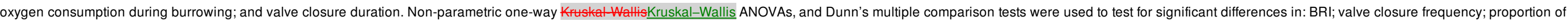
time spent closed; and oxygen consumption after valve closure. All values are presented as the meantthe standard error of the mean.

\section{Results}

3.1 Lake Femperaturetemperature 


\section{elsevier_TB_1516}

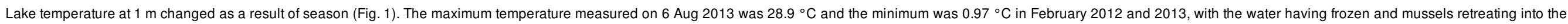

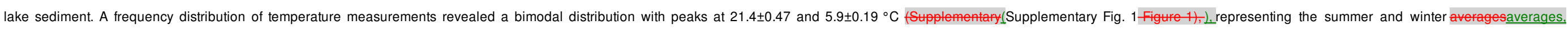

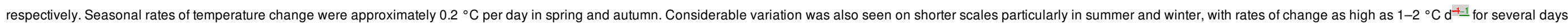
running, although diel variation was low.

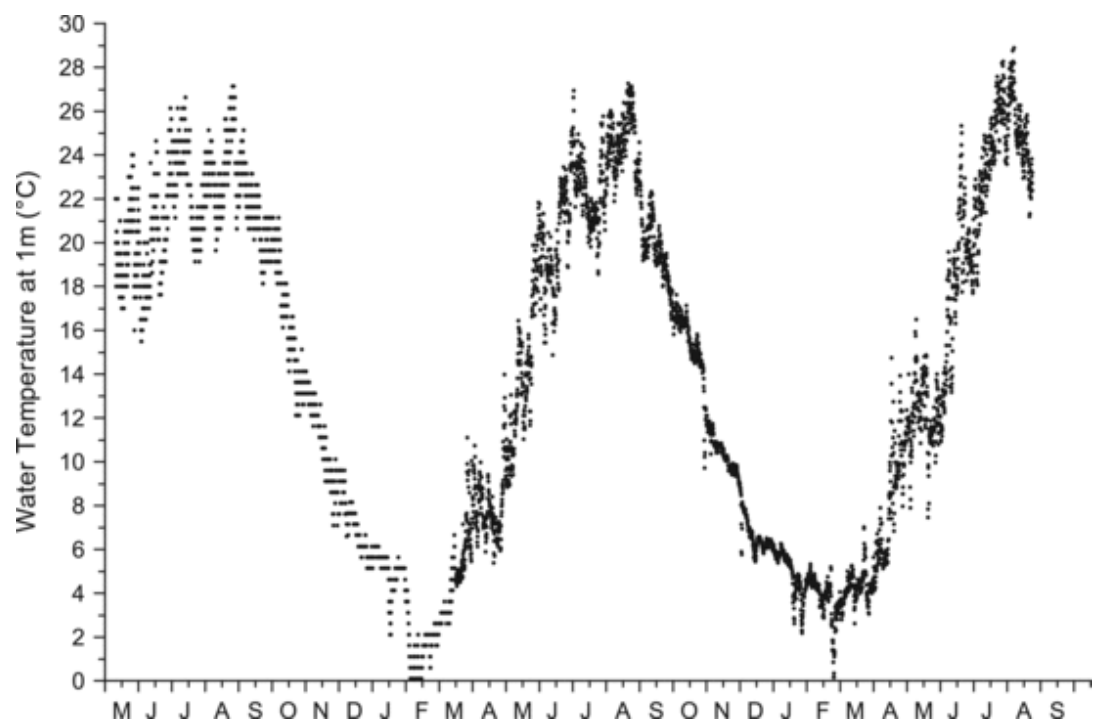

Fig. 1 Seasonal changes in water temperature in Lake Murten (Murtensee) at $1 \mathrm{~m}$ depth $\left(46^{\circ} 5424^{\prime \prime} \mathrm{N}, 7^{\circ} 259^{\circ}, \mathrm{E}\right)\left(46^{\circ} 54^{\prime} 24^{\prime \prime} \mathrm{N}, 7^{\circ} 2^{\prime} 59^{\prime \prime} \mathrm{E}\right)$ from 9 May 2011 until 23 August 2013.

\subsection{Experiment 1: Seasonal Changeschanges in Behavioutbehaviour}

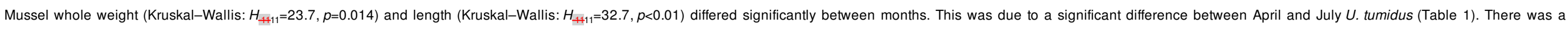

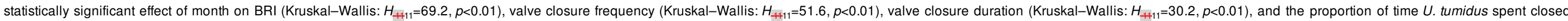

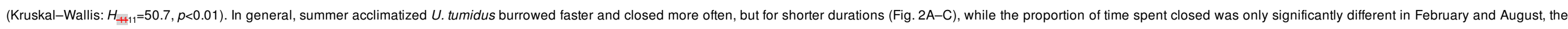

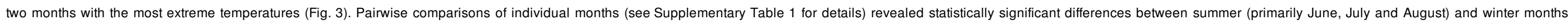

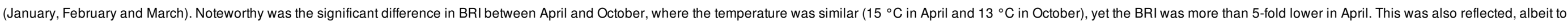
a lesser degree, in the valve closure frequency and duration, which were both more than 2-fold lower in April.

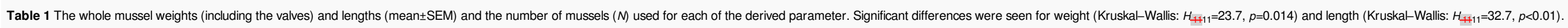

\begin{tabular}{|c|c|c|c|c|c|c|}
\hline Month & Mean Weightweight (g) & Mean tengthlength $(\mathrm{mm})$ & Observation Periodperiod (d) & $N$ (total) & Burrowing & Valve elostreclosure \\
\hline Jan & $22.0 \pm 1.2$ & $57.4 \pm 1.2$ & $2.02 \pm 0.29$ & 17 & 16 & 6 \\
\hline Feb & $21.3 \pm 2.0$ & $56.4 \pm 2.0$ & $8.32 \pm 0.91$ & 13 & 4 & 9 \\
\hline Mar & $18.0 \pm 2.6$ & $51.6 \pm 2.8$ & $2.21 \pm 0.42$ & 13 & 8 & 5 \\
\hline Apr & $14.4 \pm 2.3$ & $45.5 \pm 3.3$ & $4.24 \pm 0.55$ & 13 & 10 & 10 \\
\hline May & $20.0 \pm 3.5$ & $52.9 \pm 4.0$ & $2.04 \pm 0.24$ & 10 & 10 & 8 \\
\hline Jun & $20.9 \pm 2.4$ & $53.9 \pm 2.1$ & $0.96 \pm 0.04$ & 27 & 22 & 10 \\
\hline Jul & $27.6 \pm 2.7$ & $59.8 \pm 1.6$ & $1.60 \pm 0.20$ & 17 & 14 & 16 \\
\hline
\end{tabular}


elsevier_TB_1516

\begin{tabular}{|c|c|c|c|c|c|c|}
\hline Aug & $23.0 \pm 2.3$ & $57.9 \pm 2.1$ & $2.52 \pm 0.61$ & 14 & 11 & 14 \\
\hline Sep & $21.2 \pm 2.5$ & $56.6 \pm 2.3$ & $2.76 \pm 0.55$ & 13 & 13 & 6 \\
\hline Oct & $24.3 \pm 2.2$ & $60.4 \pm 1.9$ & $1.08 \pm 0.34$ & 16 & 16 & 6 \\
\hline Nov & $28.0 \pm 3.5$ & $59.1 \pm 2.1$ & $2.78 \pm 0.86$ & 16 & 11 & 5 \\
\hline Dec & $16.3 \pm 2.1$ & $47.6 \pm 2.6$ & $1.15 \pm 0.10$ & 16 & 12 & 7 \\
\hline
\end{tabular}
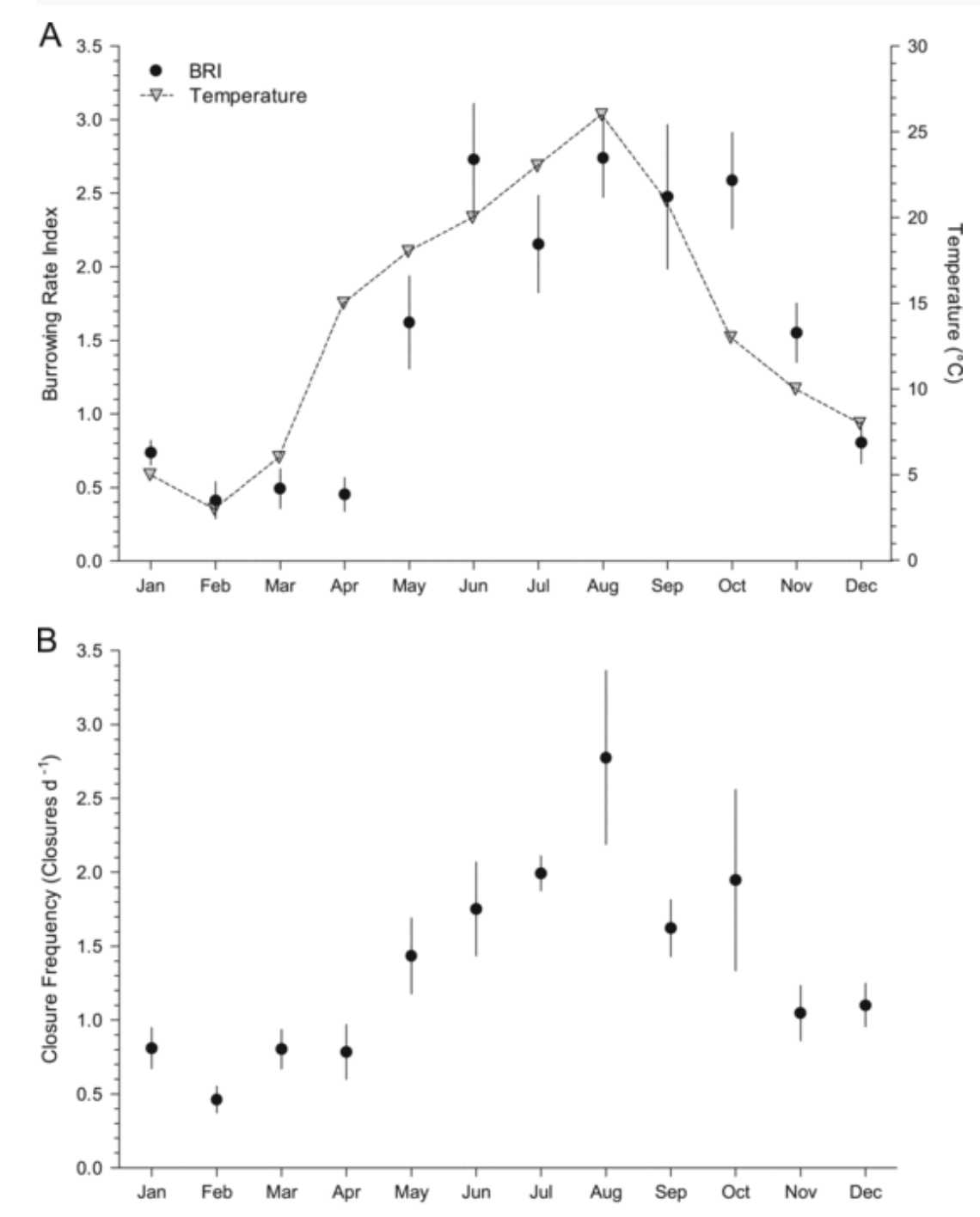

C

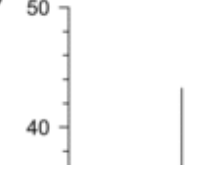




\section{elsevier_TB_1516}

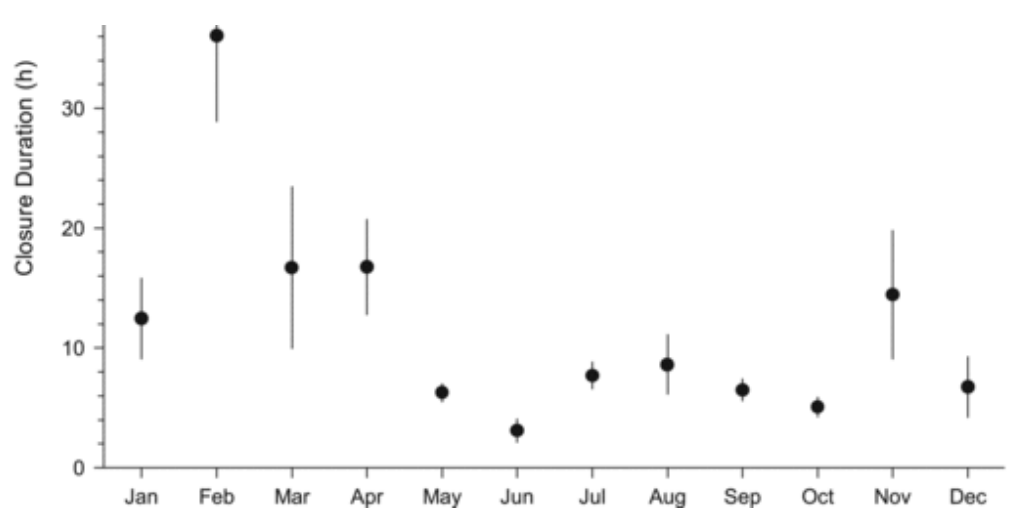

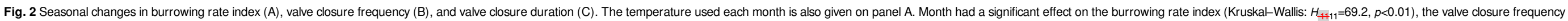

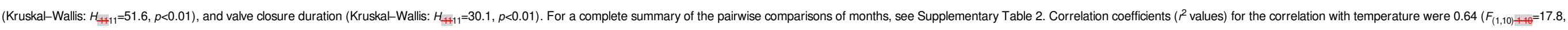
$p<0.01)$ for burrowing rate index, $0.73\left(F_{(1,10)+110}=27.1, p<0.01\right)$ for valve closure frequency, and $0.39\left(F_{(1,10) 1+10}=6.27, p=0.03\right)$ for valve closure duration.

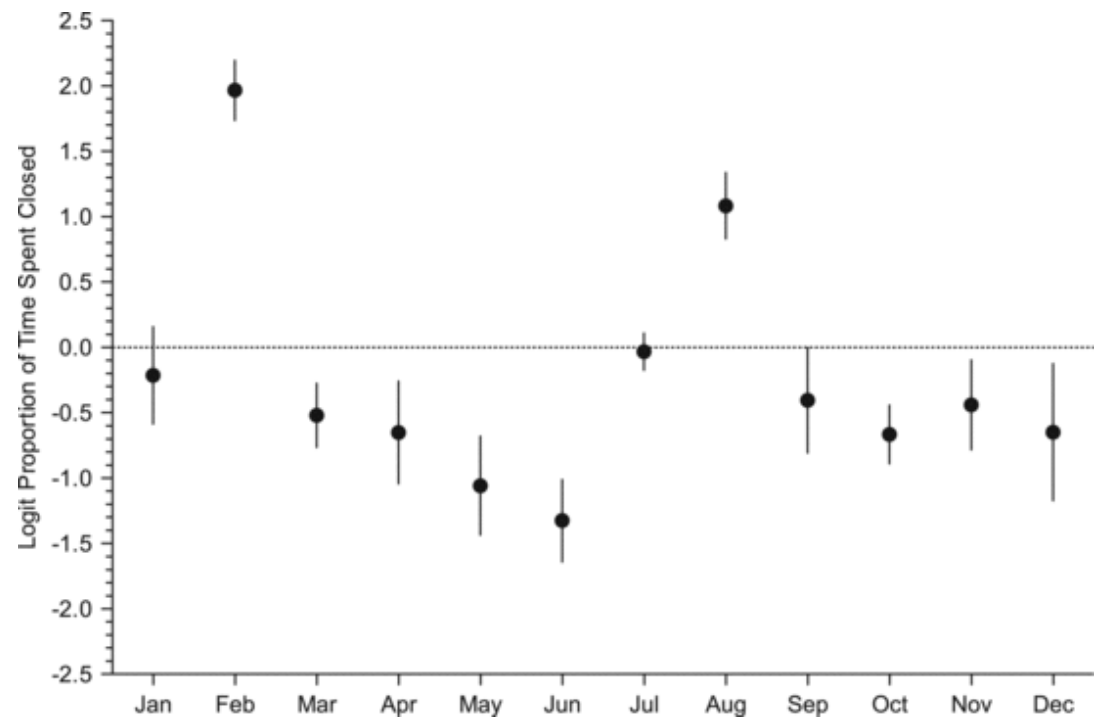

Fig. 3 Seasonal changes in the proportion of time Unio tumidus spent closed. The temperature used each month is also given in Fig. 2A. Month had a significant effect on the proportion of time spent closed (Kruskal-Wallis: $\left.H_{ \pm-11}=50.7, p<0.01\right)$.

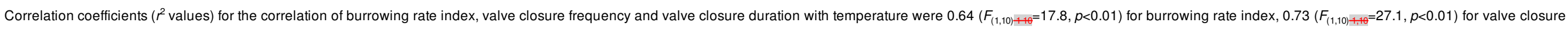

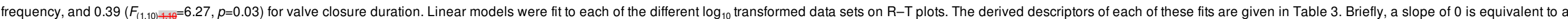

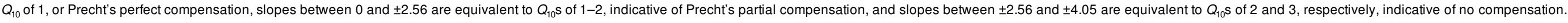
For the BRI, the slope approximated an idealized R-T plot slope indicative of non-compensation, while the valve closure frequency and duration slope values were closer to an idealized R-T plot slope indicative of partial compensation.

Table 2 Morphometric data from mussels used for the determination of oxygen consumption in experiment 2. Whole mussel weight includes the valves. Values are means $\mathrm{SEM}$.

\begin{tabular}{|c|c|c|c|c|}
\hline Parameter & Summer & Autumn & Winter & Spring \\
\hline Whole mussel weight (g) & $19.3 \pm 2.06$ & $17.4 \pm 1.50$ & $17.3 \pm 2.22$ & $20.4 \pm 0.51$ \\
\hline Length (mm) & $53.1 \pm 2.03$ & $52.3 \pm 1.58$ & $51.1 \pm 2.13$ & $56.1 \pm 0.67$ \\
\hline
\end{tabular}


Table 3 Descriptive parameters (mean \pm SEM) derived from the linear model fits to $\mathrm{R}-\mathrm{TR}-\mathrm{T}$ transformed data of rate $\left(\log _{10}\right.$ of rate) versus temperature (1000/K) from experiment 1.

\begin{tabular}{|c|c|c|c|c|}
\hline Rate & Slope & Intercept & $p$ P valtue $\underline{\underline{y} \text { alue }}$ & $+r^{2}$ value \\
\hline BRI & $-2.63 \pm 0.78$ & $9.23 \pm 2.7$ & 0.007 & 0.53 \\
\hline Valve closure frequency (closures $\mathrm{d}^{-11}$ ) & $-1.92 \pm 0.44$ & $6.77 \pm 1.5$ & 0.001 & 0.66 \\
\hline Valve closure duration (h) & $1.89 \pm 0.63$ & $-5.73 \pm 2.2$ & 0.013 & 0.48 \\
\hline
\end{tabular}

\subsection{Experiment 2: Energetic Costcost of Burrowing burrowing and Valve Closurevalve closure}

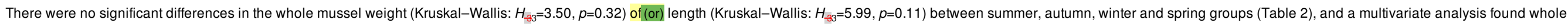

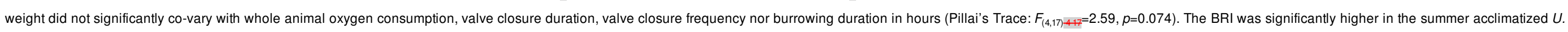

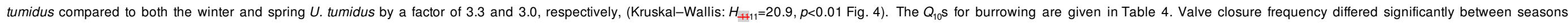

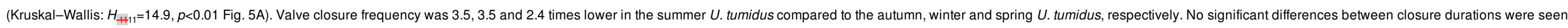

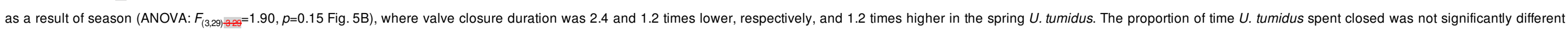
between groups (Kruskal-Wallis: $H_{1+11}=4.90, p<0.18$ ).

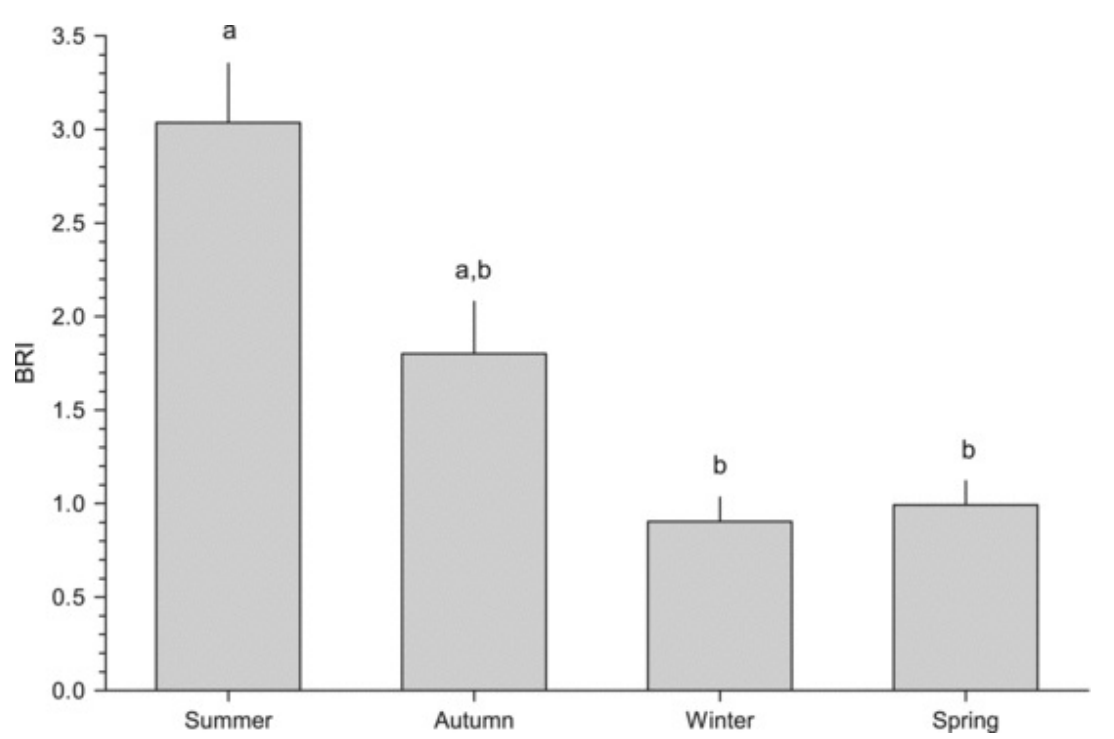

Fig. 4 The burrowing rate index $(\mathrm{BRI})$ in summer $\left(24^{\circ} \mathrm{C}\right)$, autumn $\left(14^{\circ} \mathrm{C}\right)$, winter $\left(4^{\circ} \mathrm{C}\right)$ and spring $\left(14^{\circ} \mathrm{C}\right)$ acclimatized Unio tumidus. Significant differences between seasons (where $\left.p<0.05\right)$ are indicated by different letters.

Table 4 The $Q_{10}$ values for burrowing, valve closure and oxygen consumption from experiment 2 .

\begin{tabular}{|c|c|c|c|c|c|c|}
\hline$Q Q_{10}$ values & BRI & Closure frequency & Closure duration & $\mathrm{MO}_{2}$-burrowing & $\mathrm{MO}_{2}$-valve closure & $\mathrm{MO}_{2}$-rest \\
\hline Summer-AuttumnSummer-Autumn & 1.69 & 3.48 & 4.51 & 2.13 & 1.60 & 2.14 \\
\hline Summer-WinterSummer-Winter & 1.83 & 1.86 & 1.64 & 2.37 & 1.96 & 2.43 \\
\hline Summer-SpringSummer-Spring & 3.07 & 2.44 & 1.94 & 1.96 & 2.09 & 1.45 \\
\hline Auttum-WinterAutumn-Winter & 1.99 & 1.00 & 0.60 & 2.63 & 2.41 & 2.74 \\
\hline
\end{tabular}




\section{elsevier_TB_1516}

Spring-WinterSpring-Winter
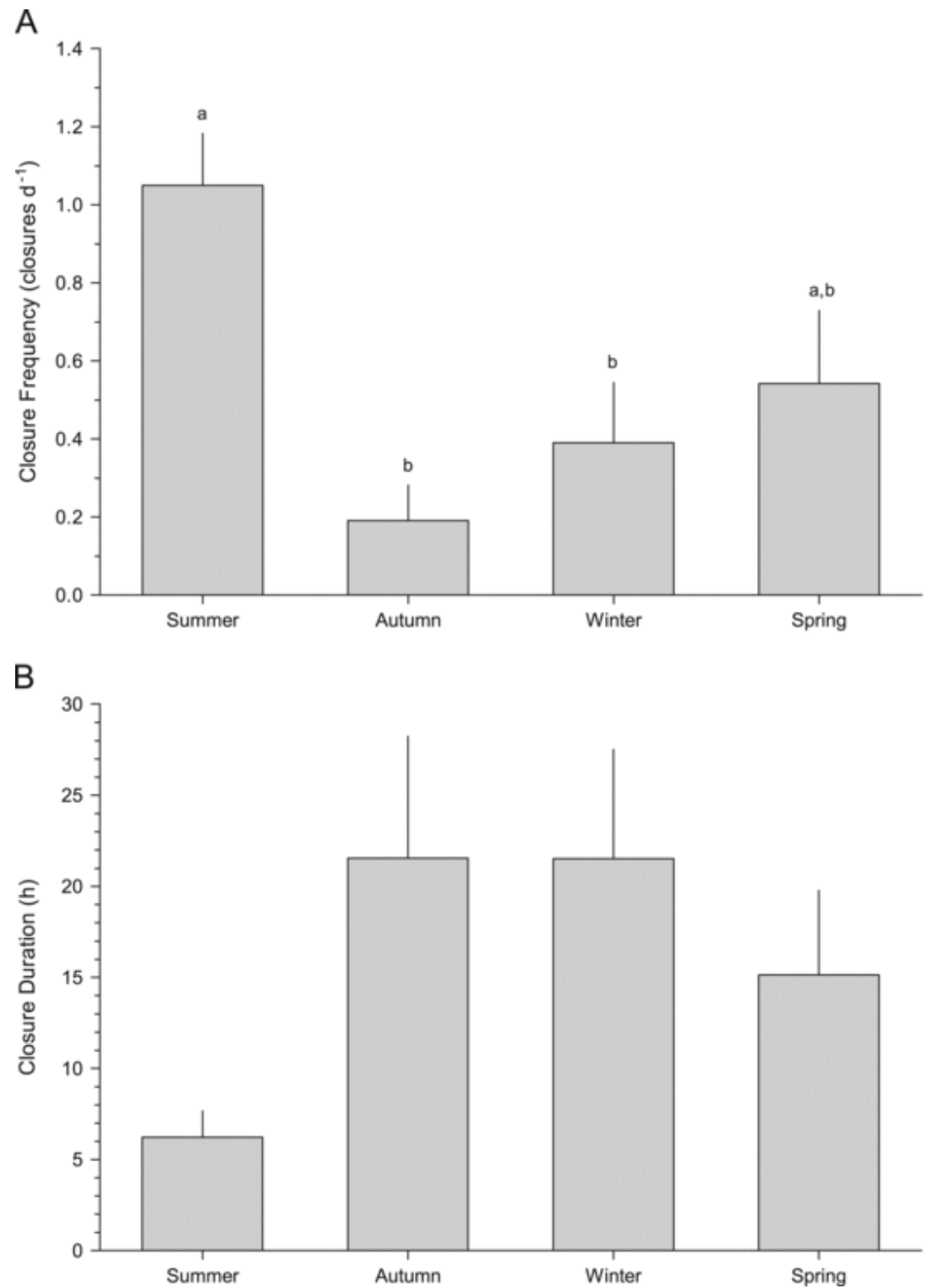

Fig. 5 The valve closure frequency $(A)$ and valve closure duration $(B)$ in summer $\left(24^{\circ} \mathrm{C}\right)$, autumn $\left(14^{\circ} \mathrm{C}\right)$, winter $\left(4^{\circ} \mathrm{C}\right)$ and spring $\left(14^{\circ} \mathrm{C}\right)$ acclimatized Unio tumidus. Significant differences between seasons (where $\left.p<0.05\right)$ are indicated by different letters.

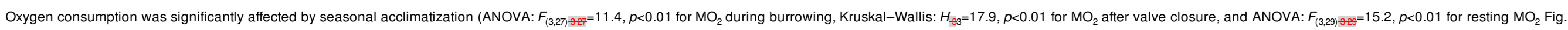

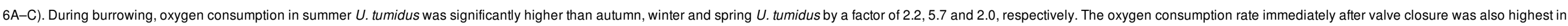
summer U. tumidus by a factor of 1.6,3.9 and 2.1 compared to autumn, winter and spring U. tumidus, respectively, however this was only statistically significant for the summer/winter U. tumidus comparison.

A 
elsevier_TB_1516

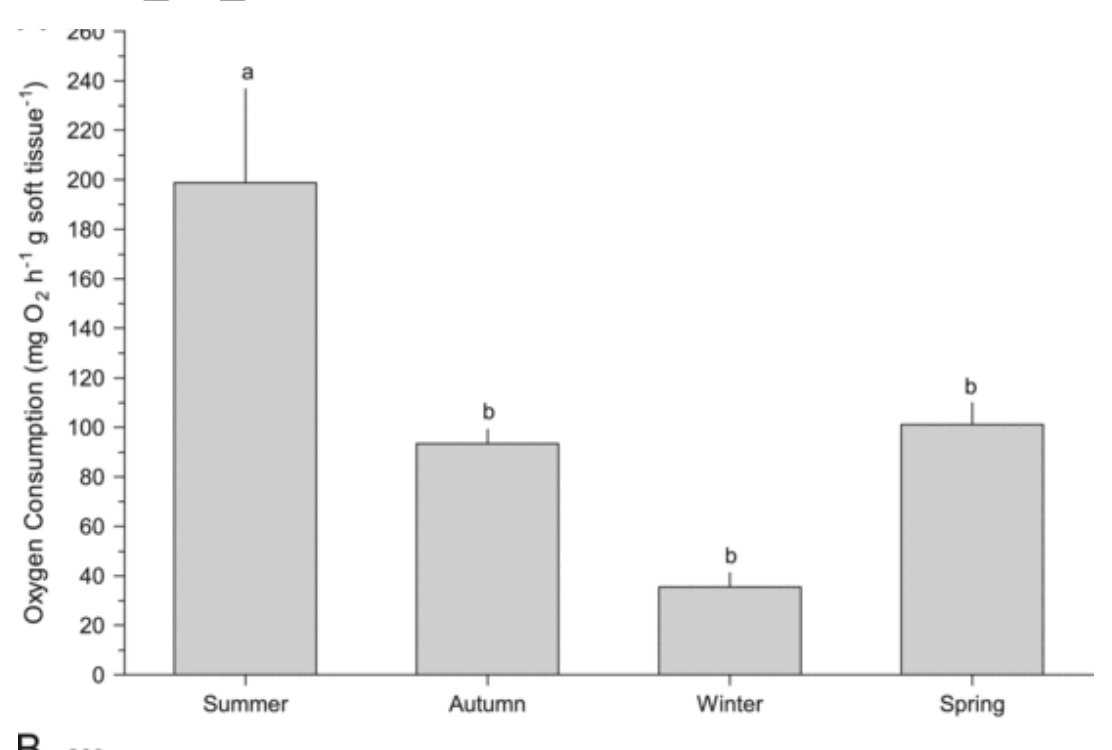

B 260

240

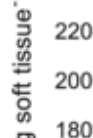

$\begin{array}{ll} & \\ \text { o } & 180 \\ \text { ¿ } 160 & \end{array}$

$\begin{array}{lll}\text { c } & 160 \\ \text { O } & 140 \\ \text { o } & 140\end{array}$

$\begin{array}{ll}\mathrm{O}^{\circ} & 140 \\ \text { E } & 120 \\ \text { ᄃ } & 20\end{array}$

흘 100

$\begin{array}{ll}\text { 总 } & 80 \\ \text { ठํ. } & 60\end{array}$

Ф.

ơ

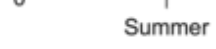

Winter

C 260

240

资 220

号 $200-$

o 180

ז 160 -

O 140

$\begin{array}{ll}\text { 을 } & 140 \\ \text { 들 } & 120- \\ \text { 을 } 100-1\end{array}$ 


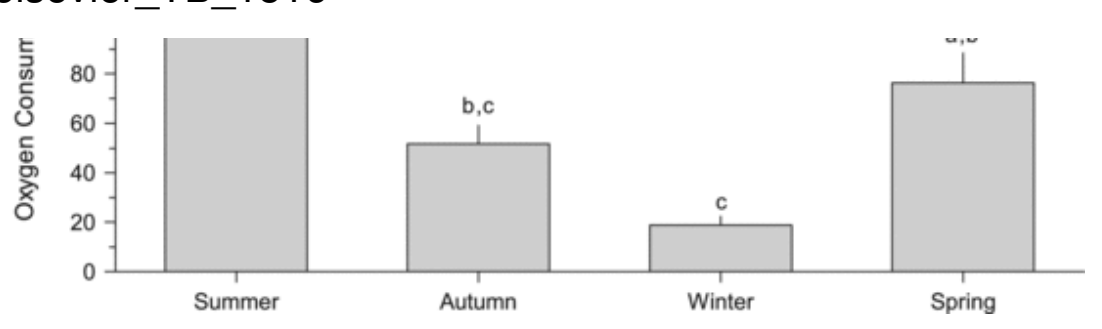

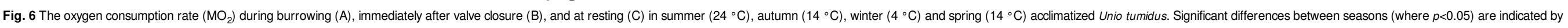
different letters.

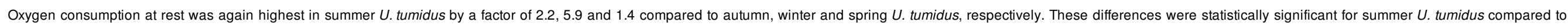

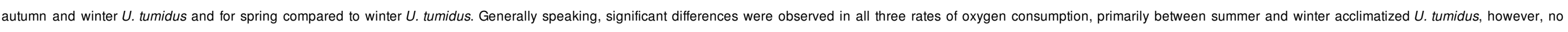
statistically significant differences were seen between autumn and spring acclimatized U. tumidus.

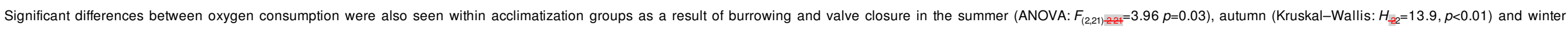

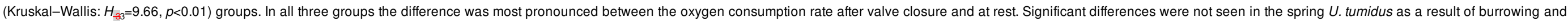

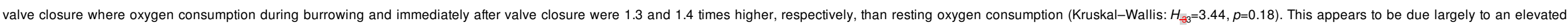
resting oxygen consumption rate.

\section{Discussion}

\subsection{Thermal Gompensationcompensation}

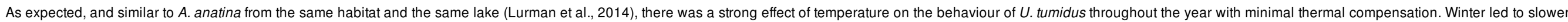

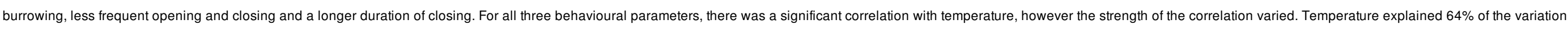

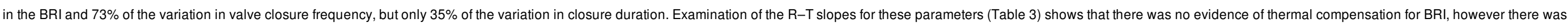

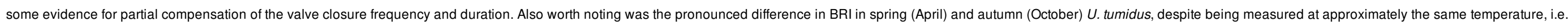
15 and $13^{\circ} \mathrm{C}$, respectively. This significant difference provoked an examination of the oxygen consumption during burrowing in spring and autumn U. tumidus in experiment 2 .

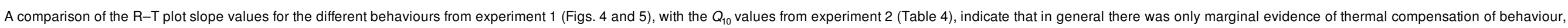

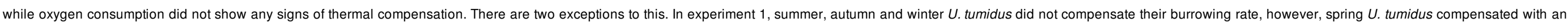

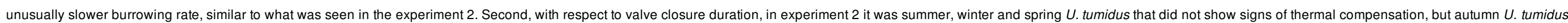
had a longer closure duration and a lower frequency, indeed even lower than winter U. tumidus.

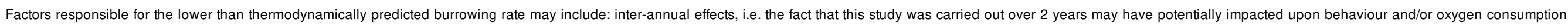

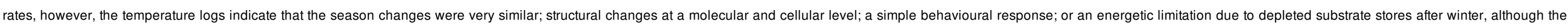

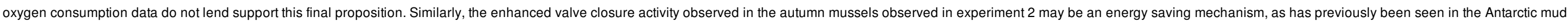

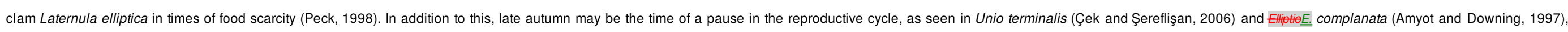
resulting in decreased valve activity.

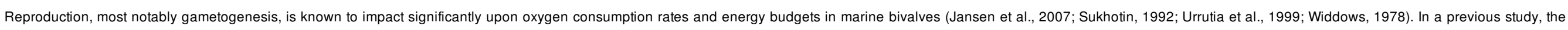

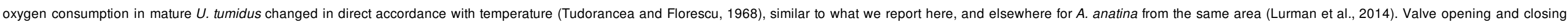

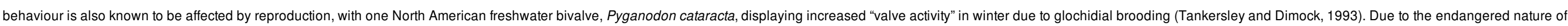

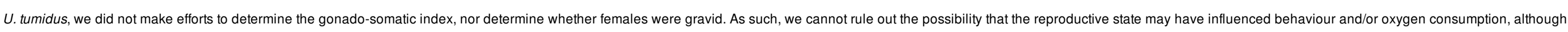




\section{elsevier_TB_1516}

we see no evidence indicating that it did.

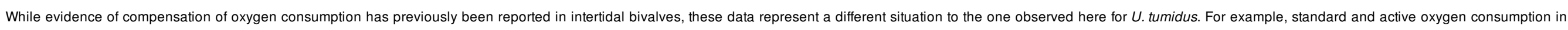

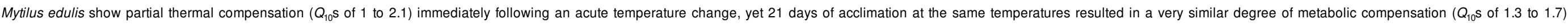

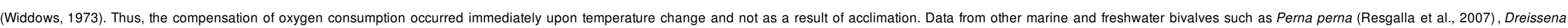

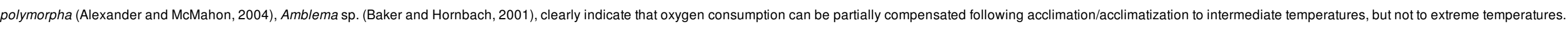

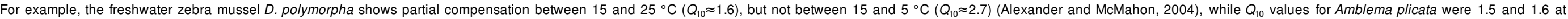

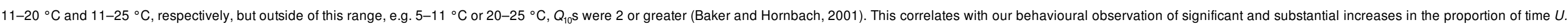

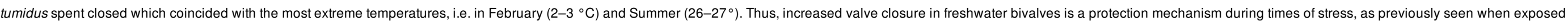
to acutely elevated temperatures (Rodland et al., 2009), or adverse conditions such as low water oxygen concentration (Chen et al., 2001), or low pH (Pynnönen and Huebner, 1995).

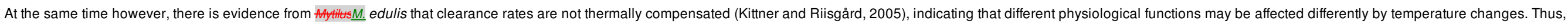

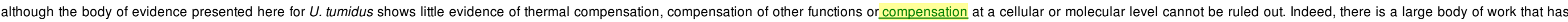

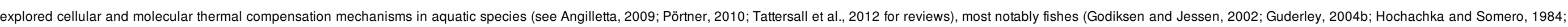

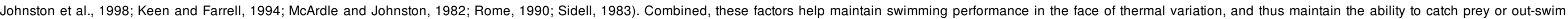

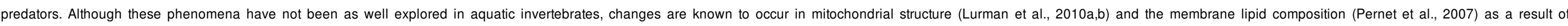

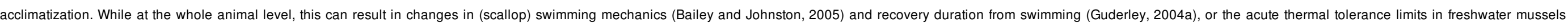
(Galbraith et al., 2012).

\subsection{Ecological Signifieaneesignificance of Not Compensatingnot compensating}

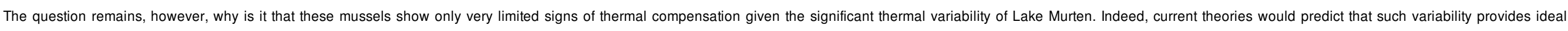

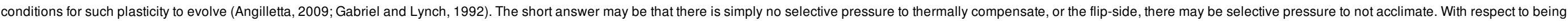

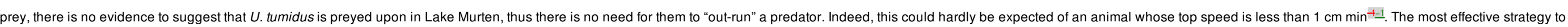

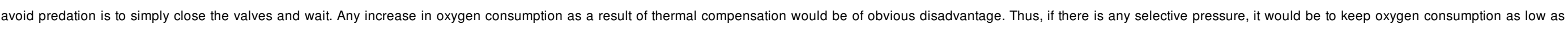

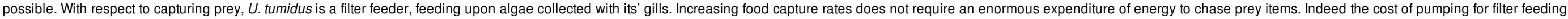

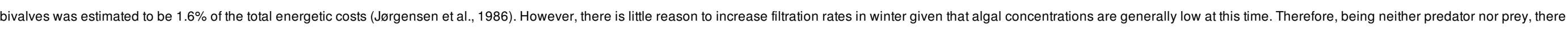
is little for $U$. tumidus to gain by increasing locomotory ability, because the associated increase in oxygen consumption rate would impinge upon their ability to close their valves when threatened.

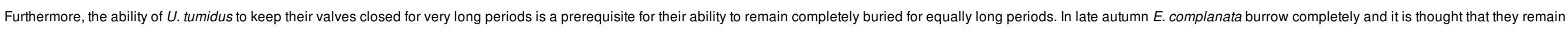

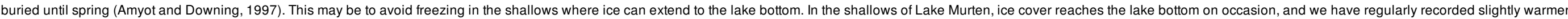

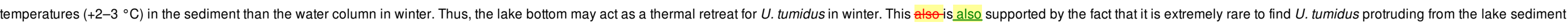

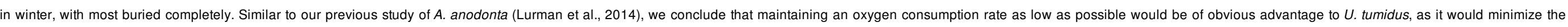
frequency and/or duration with which they would need to open their valves to respire. Thus, in many respects the costs of thermal compensation, i.e. acclimation/acclimatization, must outweigh the benefits in this low-energy species.

\section{Acknowledgements:Acknowledgements}

Christoph Lehmann is thanked for technical assistance constructing the respiration chamber. This work was supported by the University of Bern

Appendix A. Supporting information

Supplementary data associated with this article can be found in the online version at doi:10.1016/.j.jtherbio.2014.04.005.

\section{References}




\section{elsevier_TB_1516}

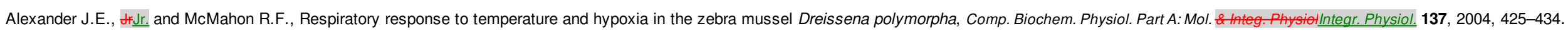

Amyot J.-P. and Downing J., Seasonal variation in vertical and horizontal movement of the freshwater bivalve Elliptio complanata (Mollusca: Unionidae), Freshwat. Bioffreshwater Biol. 37, $1997,345-354$.

Amyot J.P. and Downing J.A., Locomotion in Elliptio complanata (Mollusca: Unionidae): a reproductive function?, Freshwat. Biolfreshwater Biol. 39, $1998,351$.

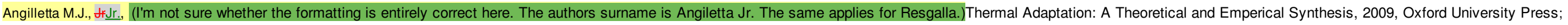
Oxford.

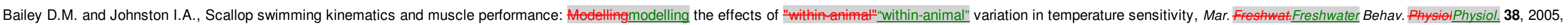
$1-19$.

Baker S.M. and Hornbach D.J., Seasonal metabolism and biochemical composition of two unionid mussels, Actinonaias ligamentina and Amblema plicata, J. Molluscan StudStud. 67, $2001,407-416$.

Catalán I.A., Johnston I.A. and Olivar M.P., Seasonal differences in muscle fibre recruitment of pilchard larvae in the north-western Mediterranean, J. Fish Biol. 64, 2004, $1605-1616$.

Çek Ş. and Şereflişan H., Certain reproductive characteristics of the freshwater mussel Unio terminalis delicates (Lea, 1863) in Gölbaşı Lake, Turkey, Aquacult. Res. 37, 2006, 1305-1315.

Chen L.-Y., Heath A.G. and Neves R.J., Comparison of oxygen consumption in freshwater mussels (Unionidae) from different habitats during declining dissolved oxygen concentration, Hydrobiologia 450, $2001,209-214$.

Clemmesen B. and Jørgensen C.B., Energetic costs and efficiencies of ciliary filter feeding, Mar. Biol. 94, 1987, 445-449.

Gabriel W. and Lynch M., The selective advantage of reaction norms for environmental tolerance, J. Evol. BiolBiol. 5, 1992, 41-59.

Galbraith H.S., Blakeslee C.J. and Lellis W.A., Recent thermal history influences thermal tolerance in freshwater mussel species (Bivalvia: Unionoida), Freshwat. SeiFreshwater Sci. 31, 2012, 83-92.

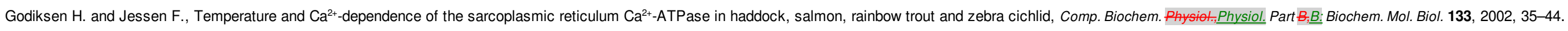

Guderley H., Locomotor performance and muscle metabolic capacities: Impact of temperature and energetic status, Comp. Biochem. Physiol,,Physiol. Part B,B: Biochem. Mol. Biol. 139, 2004a, 371-382.

Guderley H., Metabolic responses to low temperature in fish muscle, Biol. Rev. 79, 2004b, 409-427.

Hochachka P.W. and Somero G.N., Biochemical Adaptation, 1984, Oxford University Press; Oxford.

Hoffmann A.A., Acclimation: increasing survival at a cost, Trends Ecol. Evol. 10, 1995, 1-2.

Holopainen I.J., Tonn W.M. and Paszkowski C.A., Tales of two fish: the dicotomous biology of crucian carp (Carassius carassius (L.)) in northern Europe, Ann. Zool. Fenn. 34, 1997 , 1-22.

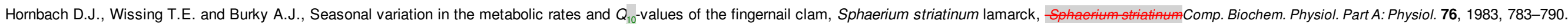

Huebner J.D., Seasonal variation in two species of unionid clams from Manitoba, Canada: respiration, Can. J. Zool. 60, 1982, 560-564.

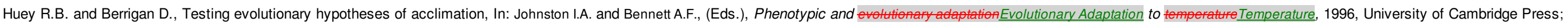

Cambridge, 205-237.

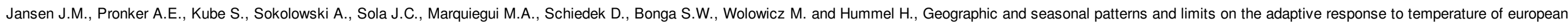

Mytilus spp. and Macoma balthica populations, Oecologia 154, 2007, 23-34.

Johnston I.A., Calvo J., Guderley H., Fernandez D. and Palmer L., Latitudinal variation in the abundance and oxidative capacities of muscle mitochondria in perciform fishes, J. Exp. Biol. 201, 1998, 1-12.

Johnston I.A. and Temple G.K., Thermal plasticity of skeletal muscle phenotype in ectothermic vertebrates and its significance for locomotory behaviour, J. Exp. Biol. 205, $2002,2305-2322$.

Jørgensen C.B., Famme P., Kristensen H.S., Larsen P.S., Møhlenberg F. and Riisgård H.U., The bivalve pump, Mar. Ecol. Prog. Ser. 34, $1986,69-77$. 


\section{elsevier_TB_1516}

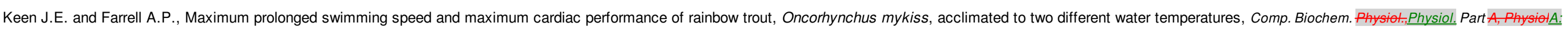
Physiol. 108, 1994, 287-295.

Kittner C. and Riisgård H.U., Effect of temperature on filtration rate in the mussel Mytilus edulis: no evidence for temperature compensation, Mar. Ecol. Prog. Ser. 305, $2005,147-152$.

Kristensen T.N., Hoffmann A.A., Overgaard J., Sørensen J.G., Hallas R. and Loeschcke V., Costs and benefits of cold acclimation in field-released Drosophila, Proc. Nat. Acad. Sci. U.S.AU.S.A. 105, $2008,216-221$.

Leroi A.M. and Bennett A.F., Temperature acclimation and competitive fitness: Anan experimental test of the beneficial acclimation assumption, Proc. Nat. Acad. Sci. ४.S.AU.S.A. 91, 1994, 1917-1921.

Lurman G., Blaser T., Lamare M., Peck L. and Morley S., Mitochondrial plasticity in brachiopod (Liothyrella spp.) smooth adductor muscle as a result of season and latitude, Mar. Biol. 157, 2010a, 907-913.

Lurman G., Blaser T., Lamare M., Tan K.-S., Poertner H., Peck L. and Morley S., Ultrastructure of pedal muscle as a function of temperature in nacellid limpets, Mar. Biol. 157, 2010b, 1705-1712.

Lurman G.J., Walter J. and Hoppeler H., Seasonal changes in the behaviour and respiration physiology of the freshwater duck mussel Anodonta anatina, J. Exp. Biol. 217, 2014, 235-243.

McArdle H.J. and Johnston I.A., Temperature adaptation and the kinetics of the $\mathrm{Ca}^{2+}$-independent and $\mathrm{Ca}^{2+}$-dependent ATPases of fish sarcoplasmic reticulum, J. Therm. Biol. 7, $1982,63-67$.

Newell R.C., Johson L.G. and Kofoed L.H., Adjustment of the components of energy balance in response to temperature change in Ostrea edulis, Oecologia 30, 1977, 97-110.

Newell R.C. and Pye V.I., The influence of thermal acclimation on the relation between oxygen consumption and temperature in Littorina littorea (L.) and Mytilus edulis L, Comp. Biochem. PhysiolPhysiol. 34, 1970 , 385-397.

Peck L.S., Feeding, metabolism and metabolic scope in Antarctic marine ectotherms, In: Pörtner H.O. and Playle R., (Eds.), Cold Ocean Physiology, 1998, Cambridge University Press; Cambridge, 365-390.

Peck L.S., Ansell A.D., Webb K.E., Hepburn L. and Burrows M., Movements and burrowing activity in the Antarctic bivalve molluscs Laternula elliptica and Yoldia eightsi, Polar Biol. 27, 2004, 357-367, (-367).

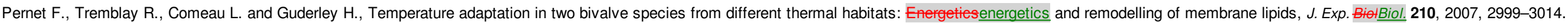

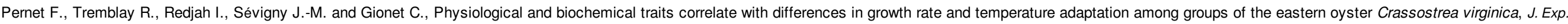
Biol. 211, 2008, 969-977.

Pörtner H.O., Oxygen- and capacity-limitation of thermal tolerance: a matrix for integrating climate-related stressor effects in marine ecosystems, J. Exp. Biol. 213, 2010, 881-893.

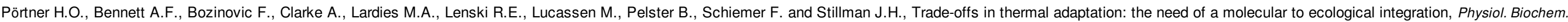
Zool. 79, 2006, 295-313.

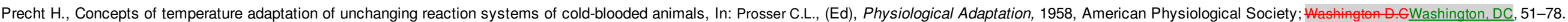

Pynnönen K.S. and Huebner J., Effects of episodic low pH exposure on the valve movements of the freshwater bivalve Anodonta cygnea L, Water Res. 29, 1995, 2579-2582.

Resgalla C., JfJr., Brasil E.d.S. and Salomao L.C., The effect of temperature and salinity on the physiological rates of the mussel Perna perna (Linnaeus 1758), Brazil. Arch. Biol. Technol. 50, $2007,543-556$.

Riascos J.M., Avalos C.M., Pacheco A.S. and Heilmayer O., Testing stress responses of the bivalve Protothaca thaca to El Nino-La Nina thermal conditions, Mar. Biol. Res. 8, $2012,654-661$.

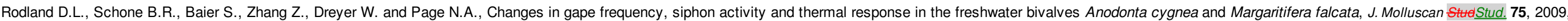
$51-57$.

Rome L.C., Influence of temperature on muscle recruitment and muscle function in vivein vivo, Am. J.Physiol, Reg.,Physiol. Reg. Int. Comp. PhysiolPhysiol. 259, 1990, R210-R222.

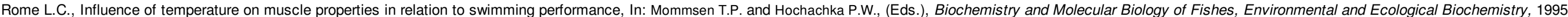
Elsevier; Amsterdam, 73-99.

Scott M., Berrigan D. and Hoffmann A.A., Costs and benefits of acclimation to elevated temperature in Trichogramma carverae, Entomol. Exp. Appl. 85, 1997, 211-219. 


\section{elsevier_TB_1516}

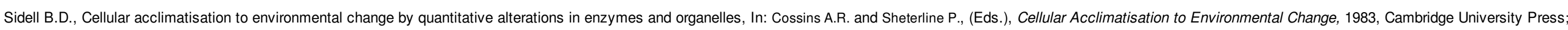
London, 103-120.

Sukhotin A.A., Respiration and energetics in mussels (Mytilus edulis L.) cultured in the White Sea, AquacutAquaculture 101, 1992, 41-57.

Tankersley R.A. and Dimock R.V., Jr, The effect of larval brooding on the respiratory physiology of the freshwater unionid mussel Pyganodon cataracta, Am. Midl. NatNat. 130, 1993, 146-163.

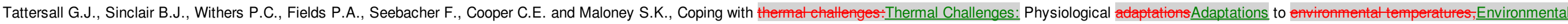
Temperatures, Comprehensive Physiology, 2012, John Wiley \& Sons, Inc.

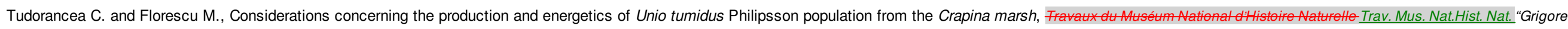
Antipa" 8, 1968, 395-405.

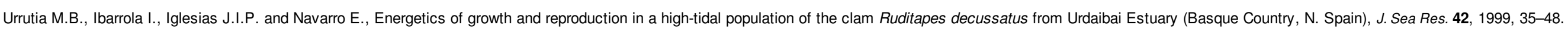
Watters G.T., A brief lookBrief Look at freshater musselFreshwater Mussel (Unionacea) biology,Biology, Freshwater Bivalve Ecotoxicology, 2007, CRC Press; Boca RatonRaton, FL, 51-64.

Widdows J., The effects of temperature on the metabolism and activity of Mytilus edulis, Neth. J. Sea Res. 7, 1973, 387-398.

Widdows J., Combined effects of body size, food concentration and season on the physiology of Mytilus edulis, J. Mar. Biol. Assoc. U.KU.K. 58, 1978, 109-124.

Wilson, R., 2007. Testing the benefits of acclimation to reproductive performance in male mosquitefish, in:mosquitofish. In: Mommsen, T.P., Walsh, P.J. (Eds.), SEB Main Meeting, Glasgow, p. S205.

Wilson R.S. and Franklin C.E., Testing the beneficial acclimation hypothesis, Trends Ecol. Evol. 17, 2002, 66-70.

Woods H.A. and Harrison J.F., Interpreting rejections of the beneficial acclimation hypothesis: Whenwhen is physiological plasticity adaptive?, EvolEvolution 56, 2002, 1863-1866.

\section{Appendix A. Supporting information}

Multimedia Component 1

Fig. S1 (i don't know if the end figure will be that small, but when I click on the link, the figure presented is no bigger than a postage stamp and impossible to read.)

Multimedia Component 2

Supplementary material

Highlights

- The freshwater mussel Unio tumidus experiences significant seasonal temperature variation.

- Burrowing rate and valve closure behaviour vary directly with temperature.

- Oxygen consumption also varies directly with temperature.

- There is little evidence of thermal compensation in Unio tumidus.

- The costs of compensation in a low-energy species like Unio tumidus must outweigh the benefits. 


\section{elsevier_TB_1516}

\section{Queries and Answers}

Query: Please confirm that given names and surnames have been identified correctly and are presented in the desired order.

Answer: This is correct.

Query: Please provide the grant number for â€œUniversity of Bernâ€ if any.

Answer: There is no grant number. This study was paid for by departmental funding

Query: Please check the page range in ref. Peck, L.S., and correct if necessary.

Answer: The pages range I originally gave, $357-367$, is correct. 